\title{
A Mixture of Lactobacillus fermentum HFY06 and Arabinoxylan Ameliorates Dextran Sulfate Sodium-Induced Acute Ulcerative Colitis in Mice
}

\author{
Fang $\mathrm{Li}^{1, *}$ \\ Hui Huang ${ }^{2, *}$ \\ Fulejia Zhu' \\ Xianrong Zhou' \\ Zhennai Yang ${ }^{3}$ \\ Xin Zhao (D) \\ 'Chongqing Collaborative Innovation \\ Center for Functional Food, Chongqing \\ Engineering Research Center of \\ Functional Food, Chongqing Engineering \\ Laboratory for Research and \\ Development of Functional Food, \\ Chongqing University of Education, \\ Chongqing, 400067, People's Republic of \\ China; ${ }^{2}$ Department of Pain Medicine, \\ The Ninth People's Hospital of \\ Chongqing, Chongqing, 400700, People's \\ Republic of China; ${ }^{3}$ Beijing Advanced \\ Innovation Center for Food Nutrition \\ and Human Health, Beijing Technology \& \\ Business University (BTBU), Beijing, \\ I00048, People's Republic of China
}

*These authors contributed equally to this work
Correspondence: Xin Zhao; Zhennai Yang Tel +86-23-6265-3650

Email zhaoxin@cque.edu.cn;

yangzhennai@th.btbu.edu.cn
Objective: Colitis is one of the main gastrointestinal diseases threatening human health. Materials and Methods: In this study, a synbiotic composed of arabinoxylan (AX) and Lactobacillus fermentum HFY06 was tested to determine its ability to relieve dextran sulfate sodium (DSS)-induced colitis.

Results: The experimental results showed that the synergistic effect of AX and L. fermentum HFY06 alleviated the weight loss of DSS-mediated colitis mice and lowered the disease activity index (DAI) score. Determination of biochemical indicators found that the synbiotic composed of AX and L. fermentum HFY06 increased the body's antioxidant capacity and reduced inflammation. The histopathological examination results showed that the colonic crypts of the mice in the model group were disordered, goblet cells were lost, and the mucous membrane was severely damaged. However, the combination of AX and L. fermentum HFY06 can significantly reverse the histopathological changes in the colon mediated by DSS. The gene expression of colon tissue was further determined, and the results showed that the synergistic effect of AX and L. fermentum HFY06 inhibited the activation of the NF$\kappa \mathrm{B}$ signaling pathway, downregulated the mRNA expression levels of nuclear factor- $\kappa \mathrm{B}-\mathrm{p} 65$

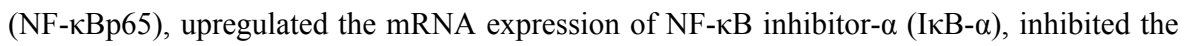
release of cytokines tumor necrosis factor- $\alpha$ (TNF- $\alpha$ ), inducible nitric oxide synthase (iNOS), and cyclooxygenase (COX-2), and exerted anti-colitis effects.

Conclusion: This study shows that the synbiotic composed of AX and L. fermentum HFY06 has the potential to prevent and treat colitis.

Keywords: Lactobacillus fermentum, arabinoxylan, colitis, inflammation, mice

\section{Introduction}

Colitis is an idiopathic, chronic inflammatory disorder. The disease usually affects the rectum, but can also involve any part of the colon. ${ }^{1,2}$ In clinical terms, chronic abdominal pain and bleeding are the most typical symptoms. The incidence rate has been increasing in the past 20 years, but its mechanism is not clear. Genetic factors, environmental factors, microbial factors, and other factors can lead to the formation and development of diseases. ${ }^{3,4}$

The intestinal microecosystem is the largest of the human body. Under normal circumstances, there are a large number of bacteria distributed in the intestine. These flora constitute a microbial barrier to provide energy and nutrition, protect the integrity of the intestinal structure, and maintain the stability of the intestinal immune system. ${ }^{5}$ Increasing evidence shows that gut microbes directly affect the 
initiation and development of colitis. ${ }^{6,7}$ For such patients, supplementation with probiotics is beneficial to the recovery and reconstruction of the intestinal flora, which in turn is beneficial for disease amelioration. There are three main methods that can be used to consume supplemental probiotics are: (i) directly consume supplemental probiotics, (ii) consume prebiotics to indirectly promote the proliferation of their own beneficial flora, or (iii) consume supplemental synbiotics, which are a combination of probiotics and prebiotics. $^{8,9}$

Probiotics are a type of active microorganism that can be orally consumed and then grown in the intestinal tract to regulate the number and types of intestinal flora, thereby further restoring the ecological balance between intestinal microorganisms. ${ }^{10}$ Probiotics are derived from food and are not pathogenic to humans, and they have high tolerance to gastric acid and bile. They can also adhere to the human intestinal mucosa and regulate the gastrointestinal flora, thereby enhancing the intestinal and system immunity. They can alleviate the symptoms of ulcerative colitis. The probiotics currently studied are mainly Bifidobacterium, ${ }^{11}$ Lactobacillus, ${ }^{12}$ and yeast. ${ }^{13}$ Probiotics are sometimes affected by the colonization ability of the flora and the survival rate of viable bacteria. Prebiotics are not digested or absorbed by the host, but can selectively promote the metabolism and proliferation of one or more beneficial bacteria in the body. ${ }^{14}$ Prebiotics can also enhance the functional activity of intestinal and bodily immune cells (macrophages, dendritic cells, T cells, $\mathrm{B}$ cells, and natural killer cells), and regulate immune cells to secrete cytokines and antibodies to exert direct immunoregulation. ${ }^{15,16}$ A study by Kangliang Shen et al found that a synbiotic composed of Bifidobacterium infantis and prebiotic xylo-oligosaccharides reduced the level of inflammation in mice with colitis, downregulated the proinflammatory cytokines TNF- $\alpha$ and IL- $1 \beta$, upregulated antiinflammatory cytokine IL-10, and also enhanced the levels of tight junction proteins ZO-1, occludin, and claudin-1 in the intestine, thereby enhancing the colonic epithelial barrier integrity. ${ }^{17}$ The results indicate that synbiotics are a promising dietary supplement or functional food. Arabinoxylan (AX), a new type of prebiotic, can specifically promote the proliferation of probiotics. Lactobacillus fermentum HFY06 is a strain that was isolated from yak yogurt that contains numerous beneficial bacteria. However, the effect of a synbiotic composed of HFY06 and AX on acute ulcerative colitis in mice is unclear.

Therefore, this study hypothesized that the synbiotics composed of HFY06 and AX can effectively alleviate acute ulcerative colitis in mice. The acute mouse colitis model induced by dextran sulfate sodium (DSS) was established by body weight analysis, serum inflammatory factor level, body oxidative stress level, colon tissue section, and colon tissue NF- $\kappa$ B signaling pathway-related gene expression to determine its possible preventive mechanism in colitis. To the best of our knowledge, this is the first study on the synbiotic composed of HFY06 and $\mathrm{AX}$ and its role and potential mechanism with regard to colitis.

\section{Materials and Methods Material/Strain Source}

L. fermentum HFY06 was isolated from a unique yak yogurt of Sichuan, China, and is stored in the China General Microbial Culture Collection, Beijing, China (No. 16636). The control strain used was Lactobacillus delbrueckii subsp. Bulgaricus (No. 1.16075). The purity of arabinoxylan (AX) was greater than 95\% (No. 120601b). The ratio of arabinose:xylose $=38 / 62$.

\section{Establishment of an Animal Model}

Fifty C57BL/6J male mice, 8 weeks old, were purchased from the Experimental Animal Center of Chongqing Medical University, Chongqing, China. The mice were randomly divided into 6 groups, namely the (i) normal group, the (ii) model group, the (iii) AX group, the (iv) HFY06 group, the (v) AX+HFY06 combined group, and the (vi) LB (Lactobacillus delbrueckii subsp. Bulgaricus) group. Among them, the LB group was used for comparison with the HFY06 group, in order to compare the antiinflammatory levels of commercial strains and L. fermentum HFY06 on high-fat diet mice. The experimental animals were adaptively fed for one week, with feeding conditions of $25 \pm 2{ }^{\circ} \mathrm{C}$, with light and dark for 12 h. Drug intervention was performed after the adaptation week (Figure 1). First, preventive intervention was performed with drugs for 7 days. During this period, all mice drank ultrapure water. The AX group was gavaged with $250 \mathrm{mg} / \mathrm{kg}$ AX polysaccharide solution, the HFY06 group was gavaged with $1 \times 10^{10} \mathrm{CFU}$ of HFY06 bacterial suspension, and $\mathrm{AX}+\mathrm{HFY} 06$ group was gavaged with $\mathrm{AX}$ polysaccharide solution $(250 \mathrm{mg} / \mathrm{kg})$ and HFY06 bacterial suspension $\left(1 \times 10^{10} \mathrm{CFU}\right)$. The normal group and model group received normal saline via intragastric administration, and the intragastric dose for all mice was $0.2 \mathrm{~mL}$. DSS induced acute colitis for 7 days. Except for the mice 


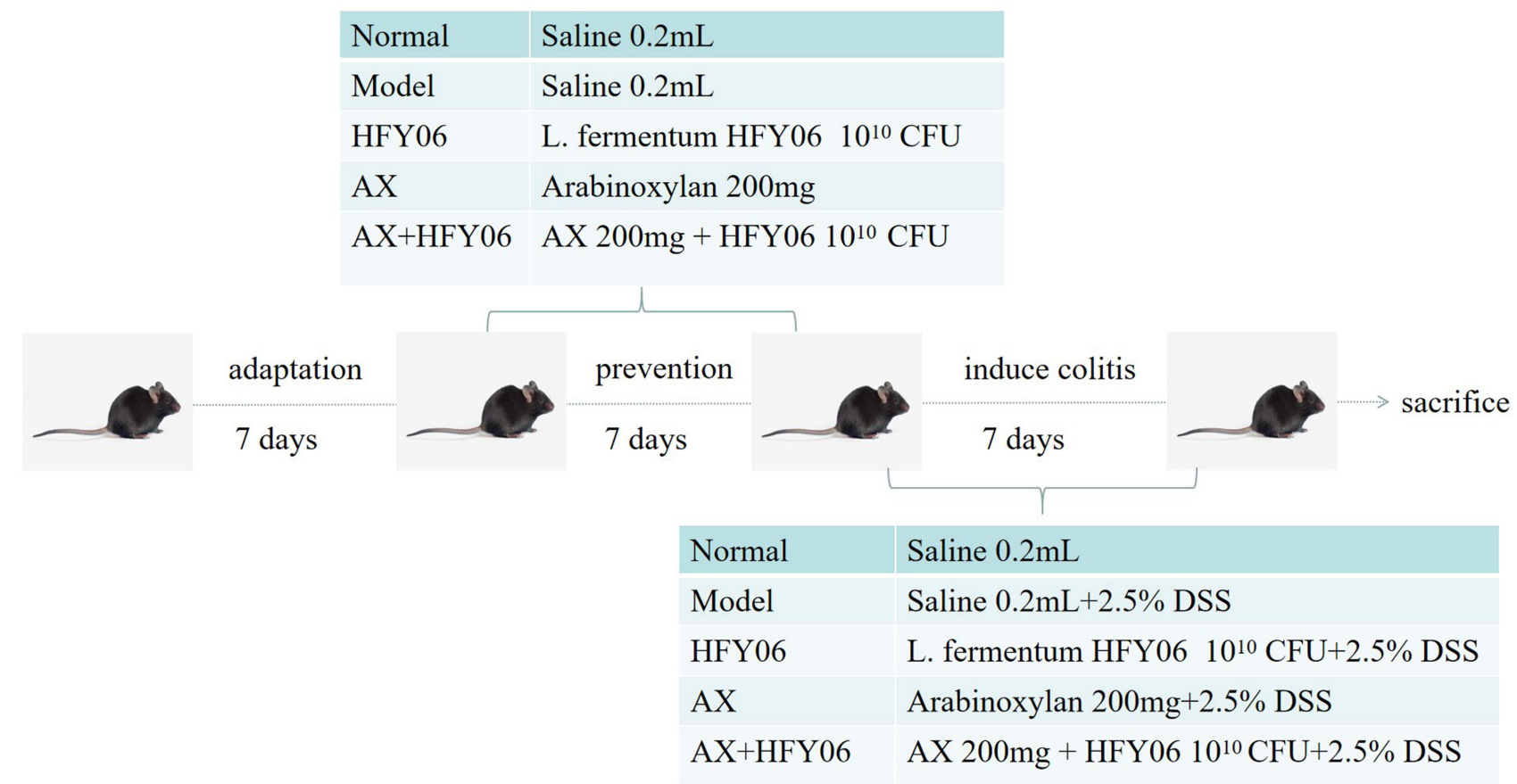

Figure I Animal experiment design.

in the normal group, the mice in the other groups drank water containing $2.5 \%$ DSS. Drug intervention was performed at the same time, and the gavage dose was consistent with the prevention period. After the last administration, the mice were fasted for $12 \mathrm{~h}$, retroorbital sinus blood collection was performed, and then, the blood was centrifuged at $3000 \mathrm{r} / \mathrm{min}$ at $4{ }^{\circ} \mathrm{C}$ for 10 $\min$. The mice were euthanized by cervical dislocation, and the colon tissue of the mice was removed. The colon tissue and serum were stored in an ultra-low temperature freezer $\left(-80^{\circ} \mathrm{C}\right)$ for further testing.

\section{Assessment of the Disease Activity Index (DAl)}

After the induction of colitis, the mice in each group were weighed daily, and their stool characteristics, as well as the condition of fecal occult blood or gross blood in the stool were recorded. Fecal occult blood detection was performed using test paper and the benzidine method: first o-diaminodimethyl biphenyl was added to the stool sample, then $2 \%$ hydrogen peroxide was added, and the color change was observed. A color change to blue indicated that the stool sample was positive for occult blood. The disease activity index (DAI) score was obtained according to Tables $1,{ }^{18}$ where DAI $=$ (weight loss score + fecal consistency score + occult/gross bleeding)/3.

\section{Measurement of Serum SOD, NO, and MDA Levels}

After thawing the collected serum, a kit (Nanjing Institute of Bioengineering, Nanjing, China) was used to determine the amounts of superoxide dismutase (SOD), nitric oxide (NO), and malondialdehyde (MDA).

Measurement of Serum IL-I $\beta$, IL-6, IL- I2, IFN- $\gamma$, TFN- $\alpha$, and IL-I0 Levels

After thawing the collected serum, a kit (Shanghai Enzyme Biotechnology Co., Ltd., Shanghai, China) was used to determine the amounts of interleukin-1 $\beta$ (IL-1 $\beta$ ), interleukin-6 (IL-6), interleukin-12 (IL-12), interferon- $\gamma$ (IFN- $\gamma$ ), tumor necrosis factor- $\alpha$ (TNF- $\alpha$ ), and interleukin-10 (IL-10) in mouse serum.

Table I Evaluation of the Disease Activity Index (DAI)

\begin{tabular}{|l|c|c|c|}
\hline $\begin{array}{l}\text { Stool } \\
\text { Consistency }\end{array}$ & $\begin{array}{c}\text { Occult/Gross } \\
\text { Bleeding }\end{array}$ & $\begin{array}{c}\text { Weight } \\
\text { Loss (\%) }\end{array}$ & Score \\
\hline Normal & Normal (-) & 0 & 0 \\
Soft but shaped & Occult blood positive (+) & $1-5$ & 1 \\
Semi loose stool & Occult blood (++) & $>5-10$ & 2 \\
Loose stool & Gross bleeding (+++) & $>10-15$ & 3 \\
Heavier loose stools & Gross bleeding(>+++) & $>15$ & 4 \\
\hline
\end{tabular}

Note: DAI = (weight loss score+fecal consistency score+occult/gross bleeding)/3. 


\section{Measurement of Colon MPO Activity}

The colon tissue was removed from storage at $-80^{\circ} \mathrm{C}$ and thawed, and the myeloperoxidase (MPO) enzymatic activity in the mouse colon tissue was measured according to the kit instructions (Nanjing Institute of Bioengineering, Nanjing, China).

\section{Pathological Examination of Colon Tissue}

Clean colon tissue without feces was removed from the newly dissected mice, and then immersed in $4 \%$ formaldehyde solution for fixation for $24 \mathrm{~h}$. Then, dehydration, embedding, sectioning, H\&E staining, and finally observing the pathological section under an optical microscope was performed, with subsequent obtainment of photographs.

\section{Determination of mRNA Expression}

The expression of iNOS, COX-2, TNF- $\alpha$, I $\kappa$ B- $\alpha$, and NF$\kappa \mathrm{Bp} 65$ in the colon tissues of the five groups of mice was measured by quantitative polymerase chain reaction assay (Q-PCR). The mouse colon tissue was removed from storage at $-80^{\circ} \mathrm{C}$, and the total RNA was extracted by the TRIzol method. A nucleic acid analyzer was used to determine the RNA concentration, and when the purity value was between 1.8-2.0, subsequent tests were then performed. The RNA was reverse transcribed into cDNA using the Thermo Scientific Reverse Transcription Kit. Finally, the reverse-transcribed cDNA was used as a template, plus fluorescent dyes, and placed in a fluorescent quantitative PCR instrument for reaction. The reaction conditions used were as follows: $95^{\circ} \mathrm{C}$ for 60 $\mathrm{s}, 40$ cycles of $95^{\circ} \mathrm{C}$ for $30 \mathrm{~s}, 65^{\circ} \mathrm{C}$ for $30 \mathrm{~s}, 72^{\circ} \mathrm{C}$ for $30 \mathrm{~s}$, then, $95^{\circ} \mathrm{C}$ for $30 \mathrm{~s}$, and $55^{\circ} \mathrm{C}$ for $35 \mathrm{~s}$, with three parallel reactions for each sample. In the experiment, the $\beta$-actin gene was used as the internal reference gene, the $-\Delta \Delta \mathrm{Ct}$ value was calculated, and the $2^{-\Delta \Delta \mathrm{Ct}}$ relative quantification method was used to calculate the expression of each group of genes. ${ }^{19}$ The primer sequences are shown in Table 2.

\section{Statistical Analysis}

The data are presented as the mean \pm standard deviation. GraphPad Prism 7 software (Graph Pad Software Inc., La
Table 2 Primer Sequence List

\begin{tabular}{|c|c|}
\hline Gene Name & Sequence \\
\hline \multirow[t]{2}{*}{ NF-кBp65 } & Forward: 5'-ATGGCAGACGATGATCCCTAC-3' \\
\hline & Reverse: 5'-CGGAATCGAAATCCCCTCTGTT-3' \\
\hline \multirow[t]{2}{*}{ IKB- $\alpha$} & Forward: 5'-TGAAGGACGAGGAGTACGAGC-3' \\
\hline & Reverse: 5'-TGCAGGAACGAGTCTCCGT-3' \\
\hline \multirow[t]{2}{*}{ iNOS } & Forward: 5'-GTTCTCAGCCCAACAATACAAGA-3' \\
\hline & Reverse: 5'-GTGGACGGGTCGATGTCAC-3'-3' \\
\hline \multirow[t]{2}{*}{ COX-2 } & Forward: 5'-GGTGCCTGGTCTGATGATG-3' \\
\hline & Reverse: 5'-TGCTGGTTTGGAATAGTTGCT-3' \\
\hline \multirow[t]{2}{*}{ TNF- $\alpha$} & Forward: 5'-CAGGCGGTGCCTATGTCTC-3' \\
\hline & Reverse: 5'-GCTGCAACAGGGGGTAACAT-3' \\
\hline \multirow[t]{2}{*}{$\beta$-actin } & Forward: 5'-CATGTACGTTGCTATCCAGGC-3' \\
\hline & Reverse: 5'-CTCCTTAATGTCACGCACGAT-3' \\
\hline
\end{tabular}

Jolla, CA, USA) was used for drawing. SPSS 17.0 software (IBM Corp., Armonk, NY, USA) was used for statistical analysis, and the groups were compared by analysis of variance (ANOVA) followed by the Duncan multi-range test $(\mathrm{p}<0.05)$.

\section{Results}

\section{Anti-Inflammatory Level of Control Strain and L. fermentum HFY06}

Table 3 shows the anti-inflammatory levels of the control strain (Lactobacillus delbrueckii subsp. Bulgaricus) and L. fermentum HFY06 on high-fat diet mice. The levels of proinflammatory factors IL-6, TNF- $\alpha$, IFN- $\gamma$ in serum of L. fermentum HFY06 mice were lower than those of control strain group mice, and the level of anti-inflammatory factor IL10 was higher than that of control strain group. It shows that L. fermentum HFY06 has a stronger anti-inflammatory level than Lactobacillus delbrueckii subsp. Bulgaricus. Therefore, this study chose L. fermentum HFY06 as the research strain, to study the lipid-lowering effect of L. fermentum HFY06 combined with arabinoxylan on high-fat diet mice.

Table 3 Comparison of Anti-Inflammatory Effects Between Control Strain and L. fermentum HFY06 Strain

\begin{tabular}{|l|c|c|c|c|}
\hline Group & IL-6 (ng/L) & IL-I 0 (ng/L) & TNF- $\alpha$ (ng/L) & IFN- $\gamma$ (ng/L) \\
\hline LB & $54.20 \pm 3.17$ & $230.16 \pm 9.36$ & $424.7 I \pm 12.82$ & $90.42 \pm 6.99$ \\
HFY06 & $50.91 \pm 2.36$ & $253.41 \pm 8.79 * * *$ & $334.21 \pm 55.45^{* *}$ & $73.58 \pm 8.13^{*}$ \\
\hline
\end{tabular}

Notes: The data are expressed as the mean \pm SD. LB: $2.5 \%$ DSS with Lactobacillus delbrueckii subsp. Bulgaricus. HFY06: $2.5 \%$ DSS with L. fermentum HFY06 (I.0 $\times 10^{10}$ CFU). $* P<0.05, * * P<0.01$, $* * * P<0.001$ compared with the LB group. 

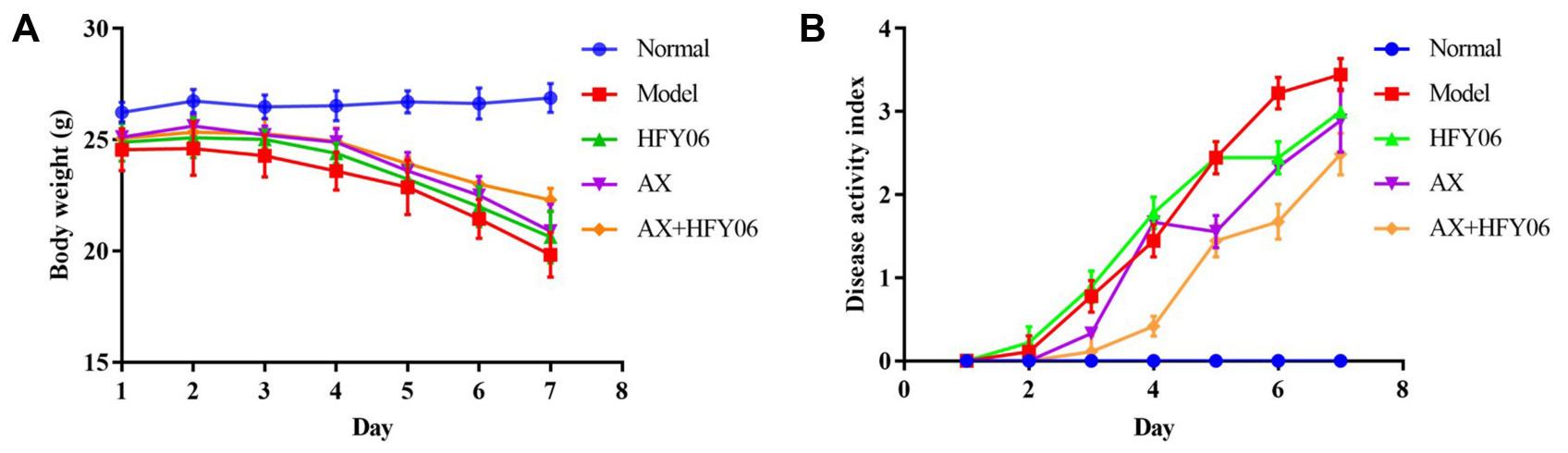

Figure 2 Effect of AX combine L. fermentum HFY06 supplementation on (A) body weight and (B) DAI of mice.

\section{Body Weight and DAI in Mice}

The weight change in mice is an indicator of the severity of colitis. As shown in Figure 2A, the body weight of mice in the normal group steadily increased, while there was a significant decrease in body weight as the modeling time increased for the mice in the model group drinking 2.5\% DSS, with weight loss beginning on the 3rd day. Some mice began to show fecal occult blood. On the 7th day, the body weight decreased to $81.56 \%$ of the original weight. After AX or L. fermentum HFY06, and AX and HFY06 synergistic intervention, the weight loss trend was alleviated. In particular, there was greater alleviation of weight loss of mice induced by DSS in the combined group of AX and HFY06, as compared to that of either AX or HFY06 alone.

As shown in Figure 2B, the DAI value of the mice in the model group reached 3.44 at 7 days. Compared with the model group, after the intervention of AX, HFY06, and AX and HFY06 combined, the DAI scores were 3, 2.89, and 2.33 , respectively, which were $12.79 \%, 15.99 \%$, and $32.27 \%$ lower than that of the model group. This shows that after treatment, the symptoms of colitis in mice abated, and a stronger effect was observed after treatment with the combination of HFY06 and AX.

\section{Serum Levels of SOD, NO, and MDA in Mice}

Compared with healthy mice, the SOD enzymatic activity in the serum of the model group mice significantly decreased, and the levels of NO and MDA significantly increased $(p<0.05)$ (Table 4$)$. There was significantly increased activity of SOD enzyme, and decreased NO and MDA in the synergistic intervention group receiving AX and HFY06, and there was a stronger effect observed with the combination of $\mathrm{AX}$ and HFY06 as compared to either AX and HFY06 alone.

\section{Serum Levels of IL-I $\beta$, IL-6, IL-I2, IFN- $\gamma$, TNF- $\alpha$, and IL- 10 in Mice}

As shown in Table 5, the levels of pro-inflammatory factors IL-1 $\beta$, IL-6, IL-12, TNF- $\alpha$, and IFN- $\gamma$ in the normal group were the lowest, and the levels of antiinflammatory factor IL-10 were the highest. The model group induced by DSS showed the opposite trend. The AX group, HFY06 group, and AX and HFY06 co-treatment group exhibited decreased levels of pro-inflammatory cytokines IL-1 $\beta$, IL-6, IL-12, TNF- $\alpha$, and IFN- $\gamma$, and increased levels of anti-inflammatory cytokine IL-10. For the pro-inflammatory factor IL-6 and the antiinflammatory factor IL-10, the combined effect of AX

Table 4 Serum Levels of SOD, NO and MDA in Mice

\begin{tabular}{|l|c|c|c|}
\hline Group & SOD $(\mathbf{U} / \mathbf{m L})$ & NO $(\boldsymbol{\mu m o l} / \mathbf{L})$ & MDA $(\mathbf{n m o l} / \mathbf{m L})$ \\
\hline Normal & $55.69 \pm 2.66^{\mathrm{c}}$ & $6.18 \pm 0.62^{\mathrm{a}}$ & $1.51 \pm 0.17^{\mathrm{a}}$ \\
Model & $34.72 \pm 2.60^{\mathrm{a}}$ & $10.5 \pm 0.32^{\mathrm{d}}$ & $5.65 \pm 0.48^{\mathrm{d}}$ \\
HFY06 & $36.61 \pm 4.75^{\mathrm{a}}$ & $8.08 \pm 0.41^{\mathrm{c}}$ & $2.57 \pm 0.25^{\mathrm{b}}$ \\
AX & $39.24 \pm 4.41^{\mathrm{ab}}$ & $8.45 \pm 0.77^{\mathrm{c}}$ & $3.30 \pm 0.24^{\mathrm{c}}$ \\
AX+HFY06 & $44.52 \pm 6.46^{\mathrm{b}}$ & $7.19 \pm 0.18^{\mathrm{b}}$ & $1.47 \pm 0.11^{\mathrm{a}}$ \\
\hline
\end{tabular}

Notes: The data are expressed as the mean \pm SD. ${ }^{a-d}$ Values in the same column with different letter superscripts indicate significant difference $(p<0.05)$. HFY06: $2.5 \%$ DSS with $L$. fermentum HFY06 (1.0 × $\left.10^{10} \mathrm{CFU}\right)$, AX: $2.5 \%$ DSS with AX (200 mg/kg), AX+HFY06: $2.5 \%$ DSS with AX $(200 \mathrm{mg} / \mathrm{kg})$ and $L$. fermentum HFY06 (1.0 × $\left.10^{10} \mathrm{CFU}\right)$. 
Table 5 Serum Levels of IL-I $\beta$, IL-6, IL-I2, TNF- $\alpha$, IFN- $\gamma$, and IL- I0 in Mice

\begin{tabular}{|c|c|c|c|c|c|c|}
\hline Group & IL-I $\beta$ (ng/L) & IL-6 (ng/L) & IL-I 2 (ng/L) & TNF- $\alpha$ (ng/L) & IFN- $\gamma(\mathbf{n g} / \mathrm{L})$ & IL-IO (ng/L) \\
\hline Normal & $43.92 \pm 3.84^{\mathrm{a}}$ & $43.68 \pm 6.82^{\mathrm{a}}$ & $60.57 \pm 6.18^{\mathrm{a}}$ & $237.57 \pm 34.37^{\mathrm{a}}$ & $56.45 \pm \mid 4.52^{\mathrm{a}}$ & $374.07 \pm 40.38^{c}$ \\
\hline Model & $66.98 \pm 8.30^{c}$ & $64.10 \pm 5.96^{\mathrm{c}}$ & $72.32 \pm 5.06^{\mathrm{b}}$ & $536.47 \pm 34.92^{c}$ & $91.92 \pm 6.62^{c}$ & $294.78 \pm 24.36^{a}$ \\
\hline HFY06 & $5 I .49 \pm 7.6 \mathrm{I}^{\mathrm{ab}}$ & $50.91 \pm 2.36^{\mathrm{ab}}$ & $58.20 \pm 3.56^{\mathrm{a}}$ & $334.21 \pm 55.45^{\mathrm{b}}$ & $73.58 \pm 8.13^{b}$ & $253.4 I \pm 8.79^{b}$ \\
\hline$A X$ & $55.27 \pm 5.8 \mathrm{I}^{\mathrm{b}}$ & $55.11 \pm 9.00^{b}$ & $58.01 \pm 8.67^{\mathrm{a}}$ & $541.09 \pm 16.26^{c}$ & $69.72 \pm 14.18^{\mathrm{ab}}$ & $297.08 \pm 34.4 I^{b}$ \\
\hline$A X+H F Y 06$ & $48.64 \pm 5.67^{\mathrm{ab}}$ & $45.39 \pm 5.57^{\mathrm{a}}$ & $55.58 \pm 4.05^{\mathrm{a}}$ & $307.22 \pm 29.85^{\mathrm{b}}$ & $63.51 \pm 7.19^{\mathrm{ab}}$ & $264.4 \mid \pm 18.43^{\mathrm{ab}}$ \\
\hline
\end{tabular}

Notes: The data are expressed as the mean \pm SD. ${ }^{a-c}$ Values in the same column with different letter superscripts indicate significant difference $(p<0.05)$. HFY06: $2.5 \%$ DSS with L. fermentum HFY06 (1.0 × $\left.10^{10} \mathrm{CFU}\right), \mathrm{AX}: 2.5 \%$ DSS with AX (200 mg/kg), AX+HFY06: $2.5 \%$ DSS with AX (200 mg/kg) and L. fermentum HFY06 (I.0 × $\left.10^{10} \mathrm{CFU}\right)$.

and HFY06 was stronger than that of AX and HFY06 alone.

\section{Colon MPO Activity in Mice}

Figure 3 shows that compared with the normal group, after DSS induction in the model group, the MPO enzymatic activity in mouse colon tissue significantly increased ( $P$ $<0.05$ ), while the AX, HFY06, and AX and HFY06 combined treatment reduced the increase in MPO enzyme induced by DSS ( $\mathrm{P}<0.05)$, especially for the combined group of AX and HFY06.

\section{Hepatic Pathological Observation}

The results of the histopathological analysis of the colons of mice (Figure 4) showed that the colonic epithelial cells of the mice in the normal group were intact, and the goblet cells were neatly arranged. DSS destroys the integrity of the colonic mucosa tissue, the crypt structure and goblet

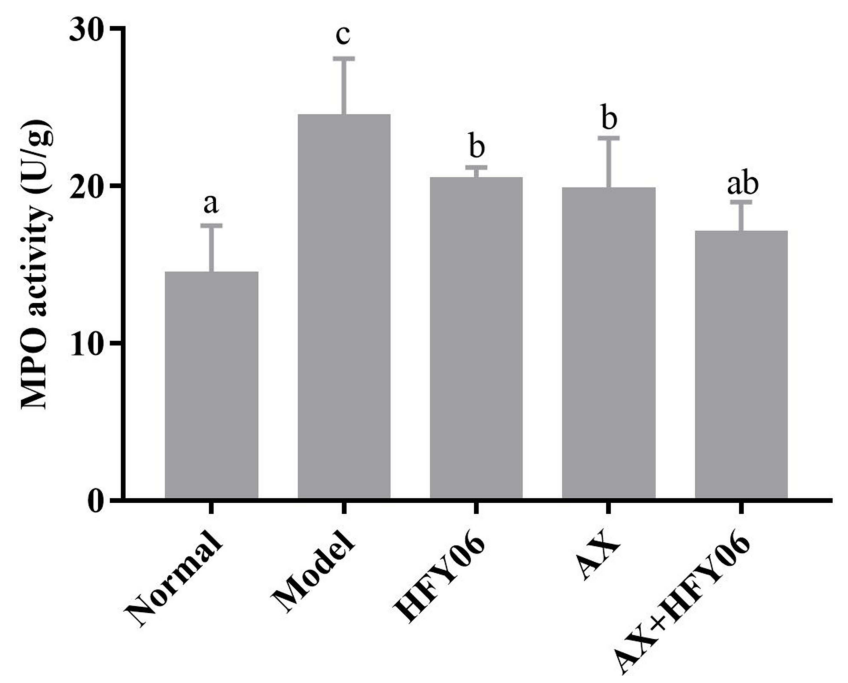

Figure 3 Effect of AX combine L. fermentum HFY06 supplementation on myeloperoxidase (MPO) enzyme activity in colon tissue. HFY06: $2.5 \%$ DSS with $L$. fermentum HFY06 (1.0 × $\left.10^{10} \mathrm{CFU}\right)$, AX: $2.5 \% D S S$ with AX (200 mg/kg), AX+HFY06: $2.5 \%$ DSS with $A X(200 \mathrm{mg} / \mathrm{kg})$ and $L$. fermentum HFY06 $\left(1.0 \times 10^{10} \mathrm{CFU}\right)$. Different letters above the histogram indicate significant differences $(P<0.05)$. cells are reduced, and there are severe ulcers and inflammatory cell infiltration in the colon. After intragastric administration of AX and HFY06, the structure of colonic epithelial cells remained destroyed, and the goblet cells were reduced, but the degree of inflammation was less than that in mice from the DSS group. In particular, the combined effect of AX and HFY06 showed that there was no obvious damage to the mouse colonic mucosa, there was an increase in goblet cells, the crypts were intact, and the integrity of the mucosal structure was effectively maintained.

\section{Liver mRNA Expression in Mice}

The NF- $\mathrm{B}$ signaling pathway controls inflammation by regulating inflammatory and anti-inflammatory factors. As shown in Figure 5, the expression level of NF$\kappa \mathrm{Bp} 65$, iNOS, COX-2, and TNF- $\alpha$ in the normal group was the lowest, and the expression level of I $\mathrm{B}-\alpha$ was the highest. After DSS induction, the expression of NF$\kappa \mathrm{Bp} 65$, iNOS, COX-2, and TNF- $\alpha$ was significantly upregulated in the model group, while the expression of IкB- $\alpha$ was downregulated $(\mathrm{p}<0.05)$. After treatment with AX, HFY06, and the AX and HFY06 synergistic blend, the expression of NF- $\kappa \mathrm{Bp} 65$, iNOS, COX-2, and TNF- $\alpha$ was inhibited, while the expression of I $\mathrm{I} B-\alpha$ was enhanced. Additionally, there was a greater combined effect of AX and HFY06 as compared to the effect of each single agent. The above results indicate that the synergistic reaction between AX and HFY06 can result in regulation of the expression of important transcription factors in the inflammatory response that inhibit inflammation, thereby alleviating local inflammation of the colon tissue.

\section{Discussion}

Colitis is an unexplained inflammatory disease of the rectum and colon. It is an uncommon inflammation with a long course and is prone to recurrence, and it occurs 


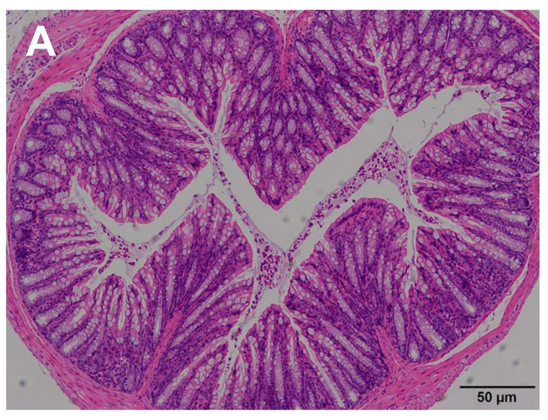

Normal

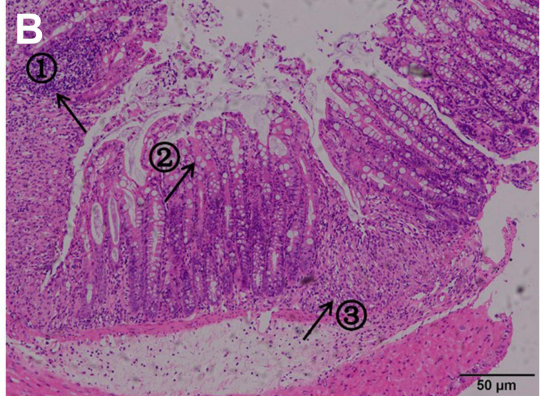

Model

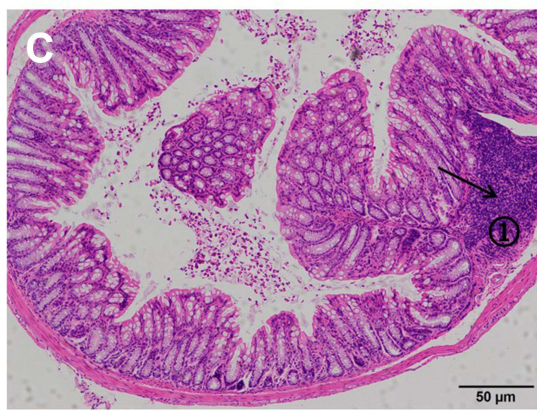

HFY06

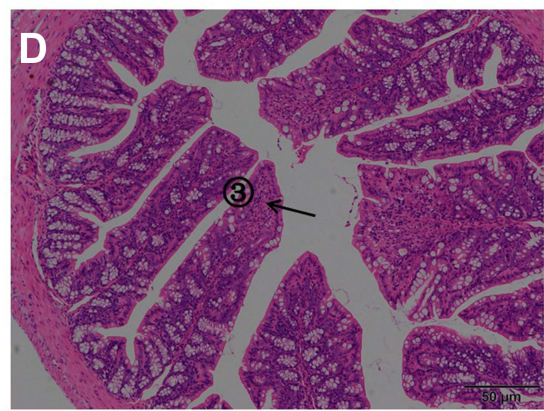

$\mathrm{AX}$

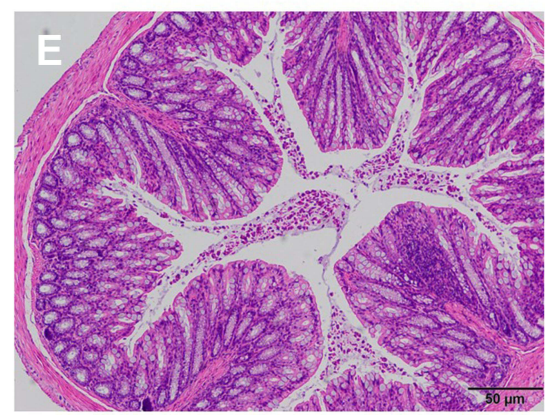

AX+HFY06

Figure 4 Effect of AX combine L. fermentum HFY06 supplementation on H\&E pathological of colon tissue in mice ((A) Normal, (B) Model, (C) L. fermentum HFY06, (D) AX, (E) L. fermentum HFY06 combined with AX). HFY06: $2.5 \%$ DSS with L. fermentum HFY06 $\left(1.0 \times 10^{10} \mathrm{CFU}\right), \mathrm{AX}: 2.5 \% \mathrm{DSS}$ with AX (200 mg/kg), AX+HFY06: $2.5 \%$ DSS with AX $(200 \mathrm{mg} / \mathrm{kg})$ and L. fermentum HFY06 ( $\left.1.0 \times 10^{10} \mathrm{CFU}\right)$. Arrow I indicates inflammatory cell infiltration, arrow 2 indicates a reduction in goblet cells, and arrow 3 indicates colonic mucosal erosion.

frequently in many countries..$^{20,21}$ It is difficult to cure with general drugs, and although many drugs have been used in the clinical treatment of colitis, they still produce side effects. Generally, patients tend to relapse after stopping the medication. Therefore, there has been great interest in safe microecological preparations with no side effects. Probiotics regulate the intestinal flora, and prebiotics provide nutritional substrates for probiotics. ${ }^{22,23}$ Studies have shown that the synbiotics formed by the combination of probiotics and prebiotics, such as those composed of Lactobacillus acidophilus, Lactobacillus rhamnosus, and inulin, can promote the balance of intestinal microecology and have a certain therapeutic effect on intestinal diseases. ${ }^{24}$ This can effectively alleviate the inflammation of the colon in mice and increase the proportion of beneficial bacteria in the intestine, but the effects of different probiotics in the treatment of colitis are very different. Liu et al found that Lactobacillus rhamnosus combined with inulin can reduce DSS-induced colitis, reduce pathological damage of colon tissue, reduce DAI score, regulate the expression of inflammatory cytokines, and have anti-inflammatory effects on mice with enteritis. ${ }^{25}$ Therefore, this study established a colitis animal test model to preliminarily explore the ability of a synbiotic composed of L. fermentum HFY06 and arabinoxylan to alleviate colitis.

In this study, we found that $\mathrm{AX}$ and L. fermentum HFY06 had significant effects on regulating mouse diarrhea, fecal occult blood, and weight loss in mice. The weight change in mice and the DAI score are two important indicators for evaluating the severity of colitis. ${ }^{26,27}$ The experimental results showed that the percentage of weight loss and the increase in the DAI score in mice mediated by DSS were significantly downregulated by AX and L. fermentum HFY06. Additionally, the synergistic effect of AX and HFY06 was greater than that of each alone. Histopathological observation revealed that DSS typically destroyed the submucosa and crypts, and the degree of inflammatory cell infiltration increased. ${ }^{28,29}$ However, there was a decrease in these pathological changes after the synergistic intervention of $A X$ and HFY06. These results suggest that the synbiotic composed of AX and HFY06 has a protective effect on DSSmediated colitis model mice. It was shown here for the first time that the synbiotic composed of AX and HFY06 exerts a strong protective effect on colitis.

The inflammatory response and oxidative stress are considered to be important pathological mechanisms that induce 
A

C
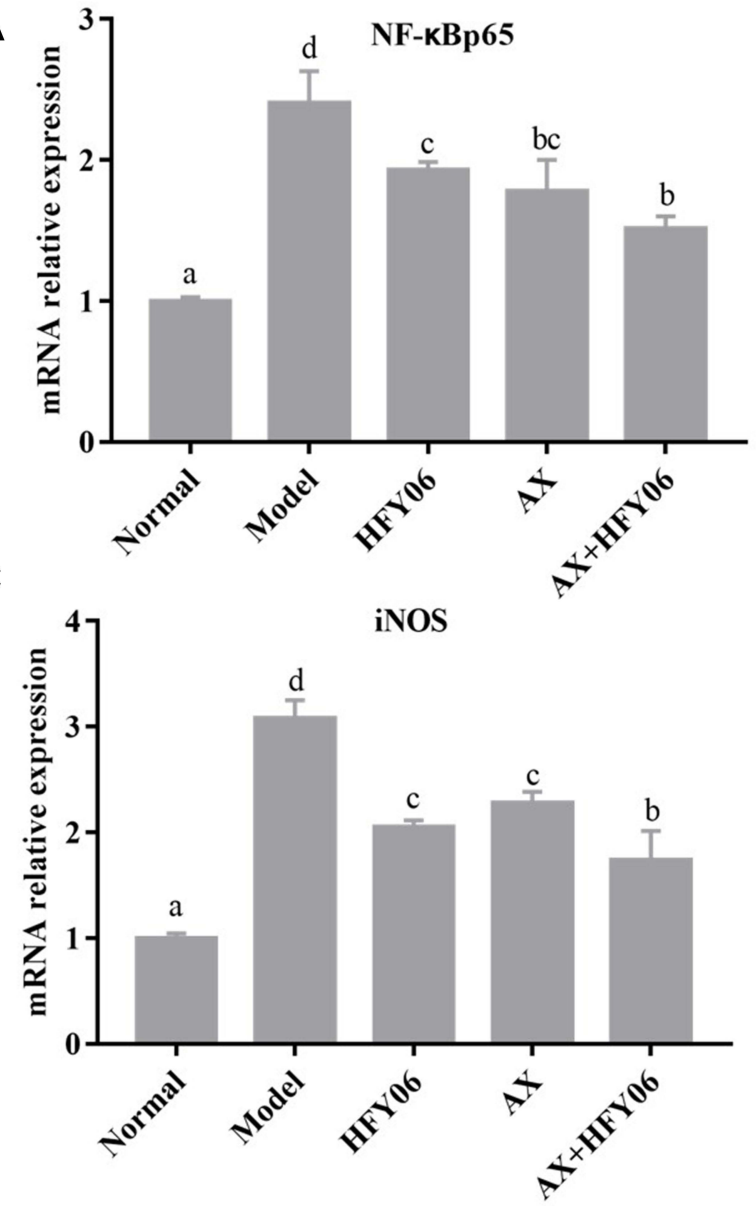

E

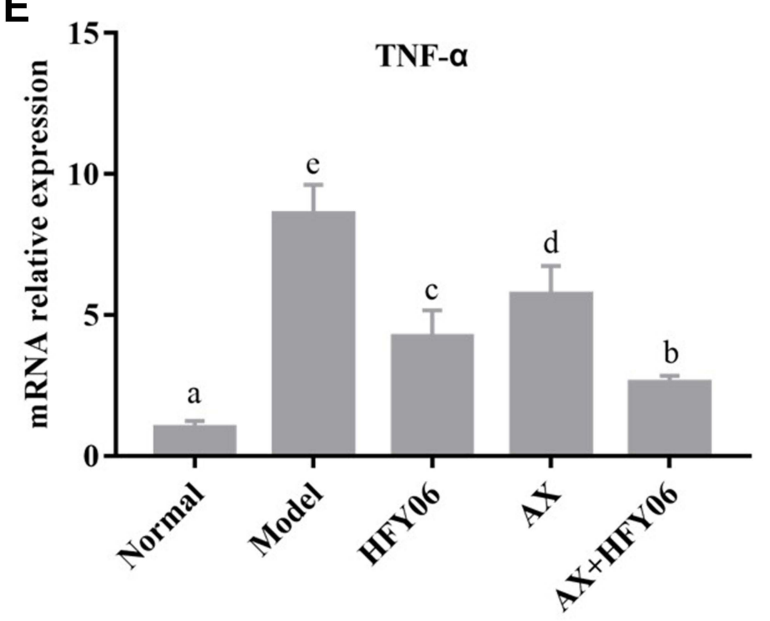

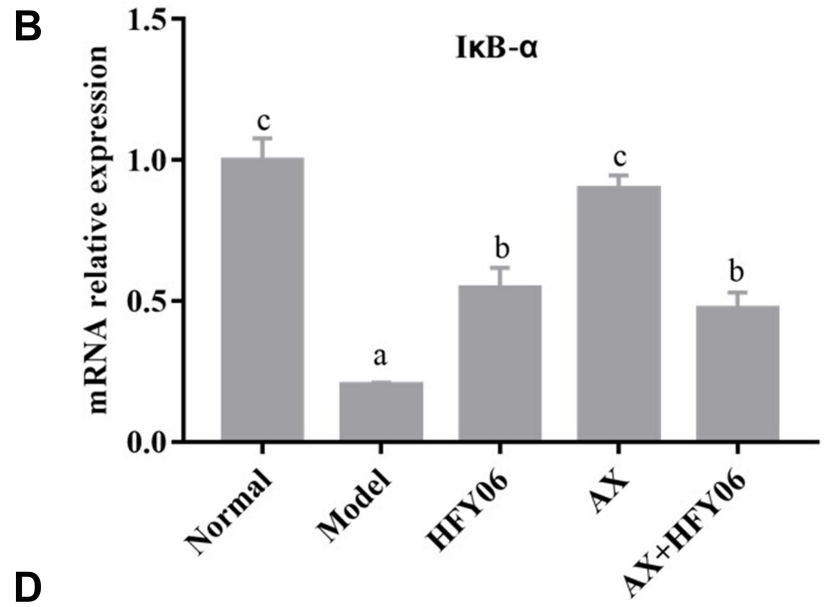

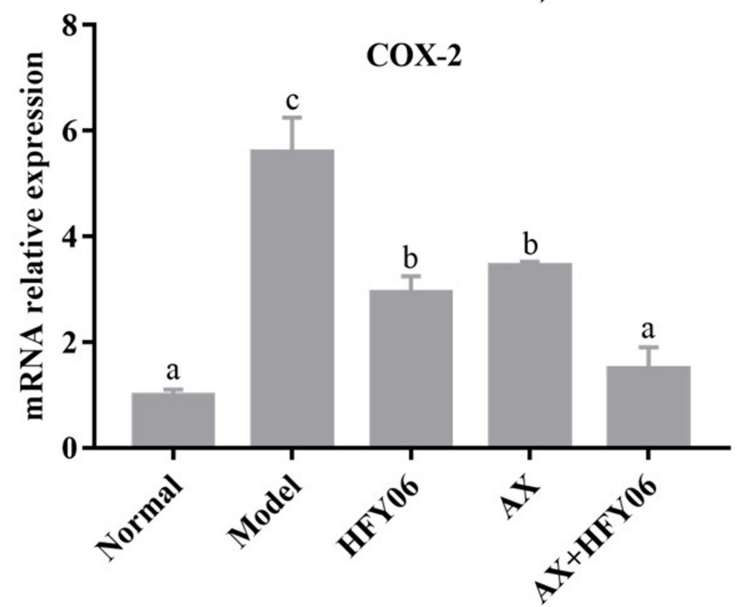

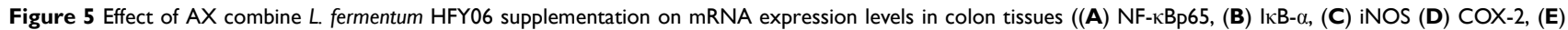
TNF- $\alpha$ ). HFY06: $2.5 \%$ DSS with L. fermentum HFY06 (I.0 × $\left.10^{10} \mathrm{CFU}\right), \mathrm{AX}: 2.5 \% \mathrm{DSS}$ with AX (200 mg/kg), AX+HFY06: $2.5 \%$ DSS with AX (200 mg/kg) and L. fermentum HFY06 $\left(1.0 \times 10^{10} \mathrm{CFU}\right)$. Different letters above the histogram indicate significant differences $(P<0.05)$.

colitis, and the two regulate and complement each other. ${ }^{30}$ Oxidative stress plays a vital role in the pathogenesis of colitis, because the body's own tissues can be attacked during the disease course. It can also activate inflammatory reactions by producing inflammatory substances through lipid peroxidation, leading to a sharp increase in inflammatory factors such as IL-6, IL-1 $\beta$, and TNF- $\alpha$, and a decrease in the level of IL-10. ${ }^{31}$ The amount of MDA can reflect the degree of tissue peroxidation damage, and the level of SOD enzymatic activity reflects the body's ability to scavenge oxygen free 
radicals. ${ }^{32}$ MPO is a glycoprotein released by neutrophils when they encounter stimuli. After neutrophils are activated, they are released outside the cell or in the phagosome to promote a series of peroxidative stress responses and accelerate local intestinal inflammation. ${ }^{33}$ The results of the current study indicate that in mice with DSS-mediated colitis, serum SOD enzymatic activity decreased, NO and MDA levels increased, and MPO enzymatic activity increased in colon tissue, indicating that DSS intervention led to the occurrence of oxidative stress in the body. At the same time, it was also observed that a large number of pro-inflammatory factors (IL-6, IL-12, IL$1 \beta$, TNF- $\alpha$, and IFN- $\gamma$ ) were produced in the serum of DSSinduced colitis mice, while the anti-inflammatory factor IL-10 decreased. After intragastric administration of $\mathrm{AX}$ and L. fermentans HFY06, inflammation and oxidative stress were alleviated, SOD enzymatic activity was significantly increased $(p<0.05)$, NO and MDA were significantly decreased $(\mathrm{p}<0.05)$, MPO enzymatic activity was significantly decreased $(\mathrm{p}<0.05)$, pro-inflammatory factors IL-6, IL- $1 \beta$, and TNF- $\alpha$ were suppressed, and anti-inflammatory factor IL-10 was increased. Similar studies have also found that the synbiotic composed of Lactobacillus rhamnosus strain GG and tagatose inhibits the regulation of pro-inflammatory cytokines and prevents the initiation of the inflammatory response, reducing the content of IL-6 and TNF- $\alpha .{ }^{34}$ These results indicate that a synbiotic composed of AX and HFY06 can increase the activity of antioxidant enzymes, and decrease lipid peroxidation and the production of free radicals, thereby alleviating the body's inflammatory response.

The NF- $\mathrm{B}$ signaling pathway is a signal transduction pathway closely related to inflammation in the body. It has a regulatory effect on the expression of various pathogenic factors such as TNF- $\alpha$, IL-6, IL-12, and some chemokines and inducible enzymes in the body that can cause colitis. ${ }^{35}$ Under normal circumstances, the cells are not stimulated, and NF- $\kappa B$ binds to its inhibitory protein I $\mathrm{B}$ and is in an inactive state. ${ }^{36}$ When cells are subjected to external stimuli, such as DSS, the $\mathrm{NF}-\kappa \mathrm{B}$ signaling pathway is activated, and the rapid phosphorylation of $\mathrm{I} \kappa \mathrm{B}$ releases the bound NF- $\kappa \mathrm{Bp} 65$ protein. NF$\kappa B p 65$ is phosphorylated and transferred into the nucleus to regulate the transcription of genes in the nucleus, including IL$1 \beta$, IL-6, COX-2, iNOS, and growth factors. ${ }^{37,38}$ Many studies have shown that the NF- $\kappa \mathrm{B}$ signaling pathway plays an important role in affecting immune and inflammatory factors. ${ }^{39,40}$ Therefore, it is necessary to further explore the pathogenesis of $\mathrm{NF}-\kappa \mathrm{B}$ in colitis.

In the current study, the PCR results showed that the mRNA expression of NF- $\kappa$ Bp65 in DSS-mediated colitis mice increased, while the mRNA expression of I $\mathrm{I} \mathrm{B}-\alpha$ decreased, and the NF- $\kappa \mathrm{B}$ signaling pathway was activated. The synbiotic composed of AX and HFY06 inhibited the mRNA expression of NF- $\mathrm{Bp} 65$ and increased the mRNA expression of $\mathrm{I} \kappa \mathrm{B}-\alpha$, thereby inhibiting the activation of the $\mathrm{NF}-\kappa \mathrm{B}$ signaling pathway. In addition, because the NF- $\kappa \mathrm{B}$ pathway was inhibited, the transcription of inflammatory genes in colon tissue was affected, and the mRNA expression of TNF- $\alpha$, iNOS, and COX-2 was subsequently inhibited. This indicates that the synbiotic composed of AX and HFY06 may alleviate colitis through the $\mathrm{NF}-\kappa \mathrm{B}$ signaling pathway.

\section{Conclusion}

This study demonstrated for the first time that a synbiotic composed of AX and L. fermentum HFY06 ameliorated symptoms of DSS-induced colitis. The synergistic effect of AX and L. fermentum HFY06 inhibited the activation of the NF- $\kappa \mathrm{B}$ signaling pathway, reduced the body's inflammatory response, and increased the antioxidant capacity. This study provides an experimental basis for the study of synbiotics in the treatment of colitis, and it is necessary to conduct further research on its mechanism.

\section{Data Sharing Statement}

The original contributions presented in the study are included in the article, further inquiries can be directed to the corresponding authors.

\section{Ethical Statement}

All of the experiments were was performed according to the 2010/63/EU directive and national standard of the People's Republic of China (GB/T 35892-2018) laboratory animal guidelines for ethical review of animal welfare and institutional rules considering animal experiments. The study protocol was approved by the Ethics Committee of Chongqing Collaborative Innovation Center for Functional Food (202009125B, Chongqing, China).

\section{Funding}

This research was funded by the Chongqing University Innovation Research Group Project (CXQTP20033), the Science and Technology Project of Chongqing Education Commission (KJQN202001614) and General Program of Natural Science Foundation of Chongqing (cstc2021jcyj msxmX0070), China., China.

\section{Disclosure}

The authors report no conflicts of interest in this work. 


\section{References}

1. Rodrigues R, Guerra G, Soares J, et al. Lactobacillus rhamnosus EM1107 in goat milk matrix modulates intestinal inflammation involving NF- $\kappa$ B p65 and SOCs-1 in an acid-induced colitis model. $J$ Funct Foods. 2018;50:78-92. doi:10.1016/j.jff.2018.09.013

2. Wu M, Li P, An Y, et al. Phloretin ameliorates dextran sulfate sodium-induced ulcerative colitis in mice by regulating the gut microbiota. Pharmacol Res. 2019;150:104489. doi:10.1016/j. phrs.2019.104489

3. Bauer $\mathrm{C}$, Lehr H, Endres S. Focus on Innate Immunity in IBD Protective and Aggravating Effects of Nlrp3 Inflammasome Activation in IBD Models: influence of Genetic and Environmental Factors. Digestive Dis. 2012;30(1):82-90. doi:10.1159/000341681

4. Danese S, Sans M, Fiocchi C. Inflammatory bowel disease: the role of environmental factors. Autoimmun Rev. 2004;3:394-400. doi:10.1016/j.autrev.2004.03.002

5. Li P, Wu M, Xiong W, et al. Saikosaponin-d ameliorates dextran sulfate sodium-induced colitis by suppressing NF- $\mathrm{KB}$ activation and modulating the gut microbiota in mice. Int Immunopharmacol. 2020;81:106288. doi:10.1016/j.intimp.2020.106288

6. Chu H, Tao X, Sun Z, Hao W, Wei X. Galactooligosaccharides protects against DSS-induced murine colitis through regulating

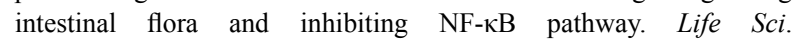
2020;242:117220. doi:10.1016/j.lfs.2019.117220

7. Walters WA, Xu Z, Knight R. Meta-analyses of human gut microbes associated with obesity and IBD. FEBS Lett. 2014;588:4223-4233. doi:10.1016/j.febslet.2014.09.039

8. Markowiak P, Ślizewska K. Effects of probiotics, prebiotics, and synbiotics on human health. Nutrients. 2017;9. doi:10.3390/nu9091021

9. Kaźmierczak-Siedlecka K, Daca A, Fic M, van de Wetering T, Folwarski M, Makarewicz W. Therapeutic methods of gut microbiota modification in colorectal cancer management-fecal microbiota transplantation, prebiotics, probiotics, and synbiotics. Gut Microbes. 2020;11:1518-1530. doi:10.1080/19490976.2020.1764309

10. Allaker RP, Stephen AS. Use of Probiotics and Oral Health. Curr Oral Health Rep. 2017;4:309-318. doi:10.1007/s40496-017-0159-6

11. Picard C, Fioramonti J, Francois A, Robinson T, Neant F, Matuchansky C. Review article: bifidobacteria as probiotic agents Physiological effects and clinical benefits. Aliment Pharmacol Ther. 2005;22:495-512. doi:10.1111/j.1365-2036.2005.02615.x

12. Muhammad I, Ahmad AA, Shah T. Health Promoting and Disease Preventing Properties of Probiotics with Special Reference to Lactobacillus: a Review. $J$ Probiotics Health. 2018;06:1-7. doi:10.4172/2329-8901.1000202

13. Lara-Hidalgo CE, Sánchez $H$, Rodríguez $D$, et al. Yeasts in Fermented Foods and their Probiotic Potential. Austin J Nutr Metab. 2017;4:1045.

14. Peredo-Lovillo A, Romero-Luna HE, Jiménez-Fernández M. Health promoting microbial metabolites produced by gut microbiota after prebiotics metabolism. Food Res Internationa. 2020;136:109473. doi:10.1016/j.foodres.2020.109473

15. Schley PD, Field CJ. The immune-enhancing effects of dietary fibres and prebiotics. Br J Nutrition. 2002;87:S221-30. doi:10.1079/BJN/2002541

16. Macfarlane GT, Macfarlane S. Fermentation in the human large intestine: its physiologic consequences and the potential contribution of prebiotics. J Clin Gastroenterol. 2011;45:S120-S127. doi:10.1097/ MCG.0b013e31822fecfe

17. Sheng K, He S, Sun M, et al. Synbiotic supplementation containing: bifidobacterium infantis and xylooligosaccharides alleviates dextran sulfate sodium-induced ulcerative colitis. Food Funct. 2020;11:3964-3974. doi:10.1039/D0FO00518E

18. Wu ZC, Zhao ZL, Deng JP. Sanhuang Shu'ai decoction alleviates DSS-induced ulcerative colitis via regulation of gut microbiota, inflammatory mediators and cytokines. Biomed Pharmacother. 2020;125:109934. doi:10.1016/j.biopha.2020.109934
19. Qian Y, Zhang J, Zhou X, et al. Lactobacillus plantarum CQPC11 isolated from Sichuan pickled cabbages antagonizes D-galactoseinduced oxidation and aging in mice. Molecules. 2018;23:1-17. doi:10.3390/molecules 23113026

20. Lim SM, Jang HM, Jeong JJ, Han MJ, Kim DH. Lactobacillus johnsonii CJLJ103 attenuates colitis and memory impairment in mice by inhibiting gut microbiota lipopolysaccharide production and NF-kB activation. $J$ Funct Foods. 2017;34:359-368. doi:10.1016/j.jff.2017.05.016

21. He D, Wang Y, Lin J, et al. Identification and characterization of alcohol-soluble components from wheat germ-apple fermented by Lactobacillus sp. capable of preventing ulcerative colitis of dextran sodium sulfate-induced mice. J Funct Foods. 2020;64:103642. doi:10.1016/j.jff.2019.103642

22. Derikx LAAP, Dieleman LA, Hoentjen F. Probiotics and prebiotics in ulcerative colitis. Best Practice Res. 2016;30:55-71. doi:10.1016/j. bpg.2016.02.005

23. Li C, Niu Z, Zou M, et al. Probiotics, prebiotics, and synbiotics regulate the intestinal microbiota differentially and restore the relative abundance of specific gut microorganisms. J Dairy Sci. 2020;103:5816-5829. doi:10.3168/jds.2019-18003

24. Verma A, Shukla G. Synbiotic (Lactobacillus rhamnosus +Lactobacillus acidophilus+inulin) attenuates oxidative stress and colonic damage in 1,2 dimethylhydrazine dihydrochloride-induced colon carcinogenesis in Sprague'Dawley rats: a long-term study. Eur $J$ Cancer Prevent. 2014;23:550-559. doi:10.1097/CEJ.00 00000000000054

25. Liu Z, Liu F, Wang W, et al. Study of the alleviation effects of a combination of: lactobacillus rhamnosus and inulin on mice with colitis. Food Funct. 2020;11:3823-3837. doi:10.1039/C9FO02992C

26. Seo S, Shin JS, Lee WS, Rhee YK, Cho CW, Hong H. Anti-colitis effect of Lactobacillus sakei K040706 via suppression of inflammatory responses in the dextran sulfate sodium-induced colitis mice model. J Funct Foods. 2017;29:256-268. doi:10.1016/j.jff.201 6.12 .045

27. Liu Y, Sheng Y, Pan Q, et al. Identification of the key physiological characteristics of: lactobacillus plantarum strains for ulcerative colitis alleviation. Food Funct. 2020;11:1279-1291. doi:10.1039/ C9FO02935D

28. Zhou X, Liu H, Zhang J, et al. Protective effect of Lactobacillus fermentum CQPC04 on dextran sulfate sodium-induced colitis in mice is associated with modulation of the nuclear factor- $\mathrm{\kappa B}$ signaling pathway. J Dairy Sci. 2019;102:9570-9585. doi:10.3168/jds.201916840

29. Watterlot L, Rochat T, Sokol H, et al. Intragastric administration of a superoxide dismutase-producing recombinant Lactobacillus casei BL23 strain attenuates DSS colitis in mice. Int J Food Microbiol. 2010;144:35-41. doi:10.1016/j.ijfoodmicro.2010.03.037

30. Wang R, Luo Y, Lu Y, et al. Maggot Extracts Alleviate Inflammation and Oxidative Stress in Acute Experimental Colitis via the Activation of Nrf2. Oxid Med Cell Longev. 2019;2019:18. doi:10.1155/2019/ 4703253

31. Amirshahrokhi K. Febuxostat attenuates ulcerative colitis by the inhibition of NF- $\mathrm{\kappa B}$, proinflammatory cytokines, and oxidative stress in mice. Int Immunopharmacol. 2019;76:105884. doi:10.1016/j. intimp.2019.105884

32. Zhao X, Song J, Yi R, et al. Comparison of antioxidative effects of insect tea and its raw tea (Kuding Tea) polyphenols in Kunming mice. Molecules. 2018;23:204. doi:10.3390/molecules23010204

33. Matuszyk A, Ceranowicz P, Warzecha Z, et al. Exogenous ghrelin accelerates the healing of acetic acid-induced colitis in rats. Int $J \mathrm{Mol}$ Sci. 2016;17:1455. doi:10.3390/ijms17091455

34. Son SJ, Koh JH, Park MR, et al. Effect of the Lactobacillus rhamnosus strain GG and tagatose as a synbiotic combination in a dextran sulfate sodium-induced colitis murine model. J Dairy Sci. 2019;102:2844-2853. doi:10.3168/jds.2018-15013 
35. Wang X, Sun Y, Zhao Y, et al. Oroxyloside prevents dextran sulfate sodium-induced experimental colitis in mice by inhibiting NF- $\mathrm{KB}$ pathway through PPAR $\gamma$ activation. Biochem Pharmacol. 2016;106:70-81. doi:10.1016/j.bcp.2016.02.019

36. Kanmani P, Kim H. Protective effects of lactic acid bacteria against TLR4 induced inflammatory response in hepatoma HepG2 cells through modulation of toll-like receptor negative regulators of mitogen-activated protein kinase and NF- $\mathrm{KB}$ signaling. Front Immunol. 2018;9:1537. doi:10.3389/fimmu.2018.01537

37. Li Q, Tian Z, Wang M, et al. Luteoloside attenuates neuroinflammation in focal cerebral ischemia in rats via regulation of the PPAR $\gamma /$ Nrf2/NF- $\mathrm{KB}$ signaling pathway. Int Immunopharmacol. 2019;66:3 09-316. doi:10.1016/j.intimp.2018.11.044
38. Hu MM, Yang Q, Zhang J, et al. TRIM38 inhibits TNF $\alpha$ - and IL-1 $\beta$ triggered NF- $\mathrm{KB}$ activation by mediating lysosome-dependent degradation of TAB2/3. Proc Natl Acad Sci U S A. 2014;111:1509-1514. doi:10.1073/pnas.1318227111

39. He R, Li Y, Han C, Lin R, Qian W, Hou X. L-Fucose ameliorates DSS-induced acute colitis via inhibiting macrophage M1 polarization and inhibiting NLRP3 inflammasome and NF-kB activation. Int Immunopharmacol. 2019;73:379-388. doi:10.1016/j.intimp.20 19.05.013

40. Dejban P, Sahraei M, Chamanara M, Dehpour A, Rashidian A. Antiinflammatory effect of amitriptyline in a rat model of acetic acid-induced colitis: the involvement of the TLR4/NF-kB signaling pathway. Fundam Clin Pharmacol. 2021;35:843-851. doi:10.1111/fcp.12642

\section{Publish your work in this journal}

The Journal of Inflammation Research is an international, peerreviewed open-access journal that welcomes laboratory and clinical findings on the molecular basis, cell biology and pharmacology of inflammation including original research, reviews, symposium reports, hypothesis formation and commentaries on: acute/chronic inflammation; mediators of inflammation; cellular processes; molecular mechanisms; pharmacology and novel anti-inflammatory drugs; clinical conditions involving inflammation. The manuscript management system is completely online and includes a very quick and fair peerreview system. Visit http://www.dovepress.com/testimonials.php to read real quotes from published authors. 